\title{
Abnormalities of localized connectivity in schizophrenia patients and their unaffected relatives: a meta-analysis of resting-state functional magnetic resonance imaging studies
}

\author{
This article was published in the following Dove Press journal: \\ Neuropsychiatric Disease and Treatment \\ 14 February 2017 \\ Number of times this article has been viewed
}

\section{Bo Xiao \\ Shuai Wang \\ Jianbo Liu \\ Tiantian Meng \\ Yuqiong $\mathrm{He}$ \\ Xuerong Luo}

Mental Health Institute of the Second Xiangya Hospital, Central South University, National Clinical Research Center on Mental Health Disorders, National Technology Institute on Mental Disorders, Hunan Key Laboratory of Psychiatry and Mental Health, Changsha, People's Republic of China
Correspondence: Xuerong Luo Mental Health Institute of the Second Xiangya Hospital, Central South University, Chinese National Clinical Research Center on Mental Health Disorders, Chinese National Technology Institute on Mental Disorders, Hunan Key Laboratory of Psychiatry and Mental Health, No 139, Middle Renmin Road, Changsha 4I00II, People's Republic of China

Tel +86 I56 75I6 4992

Email luoxr@vip.sina.com
Objective: The localized dysfunction of specialized brain regions in schizophrenia patients and their unaffected relatives has been identified in a large-scale brain network; however, evidence is inconsistent. We aimed to identify abnormalities in the localized connectivity in schizophrenia patients and their relatives by conducting a meta-analysis of regional homogeneity (ReHo) studies.

Methods: Fourteen studies on resting-state functional magnetic resonance imaging, with 316 schizophrenia patients, 342 healthy controls, and 66 unaffected relatives, were included in the meta-analysis. This analysis was performed using anisotropic effect-size-based signed differential mapping software.

Results: Schizophrenia patients showed increased ReHo in right superior frontal gyrus and right superior temporal gyrus, as well as decreased ReHo in left fusiform gyrus, left superior temporal gyrus, left postcentral gyrus, and right precentral gyrus. Unaffected relatives showed decreased ReHo in right insula and right superior temporal gyrus. These results remained widely unchanged in both sensitivity and subgroup analyses.

Conclusion: Schizophrenia patients and their unaffected relatives had extensive abnormal localized connectivity in cerebrum, especially in superior temporal gyrus, which were the potential diagnostic markers and expounded the pathophysiological hypothesis for the disorder.

Keywords: schizophrenia, localized connectivity, regional homogeneity, resting-state fMRI, meta-analysis, effect-size-based signed differential mapping

\section{Introduction}

Schizophrenia is characterized by various perceptual, cognitive, social, emotional, linguistic, and motivational disturbances that are differentially expressed in individual patients. ${ }^{1}$ Although the pathogenesis of schizophrenia has been explored in various fields such as in genetics ${ }^{2}$ and neural biochemistry, ${ }^{3}$ it is still undefined. Neuroimaging techniques have been extensively used to address the pathophysiological mechanisms of the disorder over the past decades. ${ }^{4}$ Furthermore, the abnormality of neuroimaging endophenotypes in schizophrenia is an ideal marker for exploring the relationship of neurodevelopmental abnormalities with genetic and environmental risk factors. ${ }^{5,6}$

Apart from voxel-based structural neuroimaging and task-based functional magnetic resonance imaging (fMRI) scans, task-free resting-state fMRI (rs-fMRI) studies have been successfully applied to reveal regional activity and functional connectivity in schizophrenia patients. ${ }^{7,8}$ In recent rs-fMRI studies, a regional homogeneity 
(ReHo) method was used to analyze the blood oxygen level-dependent signal in cerebrum, assume that a given voxel was temporally similar to those of its neighbors, and then finally reflect localized functional connectivity or synchronization of information processing. ${ }^{9,10}$

Previous neuroimaging studies about localized connectivity in schizophrenia patients and their unaffected relatives by ReHo analysis have revealed that several regions show abnormal synchronization compared with healthy controls. ${ }^{1-13}$ However, the evidence is inconsistent. In addition, whether schizophrenia patients and these high-risk individuals (unaffected relatives) share neuropathological characteristics remains unclear. In the present study, we conducted a metaanalysis of these ReHo studies in schizophrenia patients and their unaffected relatives and subsequently identified the changes in these endophenotypes in both individuals.

\section{Methods}

\section{Study design}

The study was designed using a voxel-based meta-analysis based on clinical data of statistical maps and peak coordinates collected from previous case-control trials of rs-fMRI regarding changes in localized connectivity in schizophrenia patients and their unaffected relatives. Two researchers (BX and JL) independently reviewed the literature and selected studies to use in the meta-analysis. Any disagreement was resolved through a group discussion.

\section{Searching strategies}

Literature searches were performed in related databases, including PubMed, Google scholar, and CNKI, before October 2016. The following search terms were combined and used: "schizophrenia/schizophrenic/SZ/SCZ", "functional MRI/fMRI", "regional homogeneity/ReHo/localized connectivity/coherence/concordance", and "relative/sibling/ twin". Publications from conferences, monographs, theses, or reference lists in identified studies were also regarded as potential sources to be included in the meta-analysis.

\section{Inclusion and exclusion criteria}

According to the Preferred Reporting Items for Systematic Reviews and Meta-Analyses guidelines, ${ }^{14}$ the following criteria were used for inclusion in the meta-analysis: 1) wholebrain analysis was used in task-free rs-fMRI studies; 2) studies included a comparison of the localized connectivity between schizophrenia patients and healthy controls or between patients and their unaffected relatives or between relatives and controls; 3 ) the coordinates (either Talairach or Montreal Neurologic Institute) of altered brain regions were detailed; and 4) the publication was in English or Chinese. Meanwhile, the following studies were excluded: 1) ROI approaches and 2) studies not performed using rs-fMRI (eg, PET, DTI, and EEG). Two researchers (BX and JL) examined the abstract or full text of all searched articles to identify studies that fit the abovementioned criteria. When multiple studies used the same patient cohort, the one with the largest sample size was selected.

\section{Data extraction and quality scores}

Two researchers (SW and TM) independently extracted data from the included studies. The general characteristics of each study, including the first author, year of publication, sample size, onset of disease, medication use, tesla of MRI, image package, full width at half maximum (FWHM), stereotactic space of coordinates, and the threshold, were extracted as the basic data. We also obtained voxel-based data, which included the peak coordinates and $t / z$ values of statistically significant differences in localized connectivity between schizophrenia patients and healthy controls or between unaffected relatives and healthy controls or between schizophrenia patients and their unaffected relatives. Missing data were acquired from the corresponding authors of the study by email. If missing data cannot be acquired, the papers were excluded. Any disagreement about the data was resolved through group discussions with consensus. All data were checked for internal consistency. The quality of the included studies was evaluated using a checklist that focused on both the clinical and demographic aspects of individual study samples and the imaging-specific methodology used in the studies. ${ }^{15,16}$ We also adopted recently established preprocessing and computational parameters. ${ }^{10,17}$

\section{Anisotropic effect size-based signed differential mapping (AES-SDM) analysis}

The meta-analysis of rs-fMRI studies was performed using AES-SDM software, which has been previously used in several neuropsychiatric disorders. ${ }^{18-20}$ AES-SDM software is a voxel-based meta-analytic approach that enabled the use of reported peak coordinates of localized functional connectivity difference in whole-brain studies. ${ }^{21}$ The SDM method has been previously described. ${ }^{22}$

Researchers BX and YH performed the meta-analysis. The main threshold was set at uncorrected $P<0.001$ (empirically equivalent to $P<0.05$, corrected $)^{19}$ with $z$ score $>1$ (peak height) and cluster extent $\geq 20$ voxels. The default settings in the AES-SDM software were used for other parameters. A leave-one-out jackknife analysis was used to determine the sensitivity of the reported results to the 
inclusion of individual studies. ${ }^{17}$ Heterogeneity among studies was assessed through the $Q$ statistic with a threshold of $P<0.05$. Subgroup analysis was performed based on disease onset (first or not) and medicated status (medicationfree or medicated).

\section{Results}

\section{Basic information of included studies}

The entire search process is as described in the QUOROMtype flowchart (Figure 1). Fourteen rs-fMRI studies on localized functional connectivity met the inclusion criteria, $13^{11,12,23-33}$ of which were involved in schizophrenia patients vs healthy controls, three ${ }^{11,13,24}$ of which were involved in unaffected relatives vs healthy controls, and one ${ }^{24}$ of which was involved in schizophrenia patients vs healthy controls. The overall sample was equivalent to a cohort of 316 schizophrenia patients, 342 healthy controls, and 66 unaffected relatives contributing data to the meta-analysis. The clinical information and quality scores of each study are detailed in Tables 1 and 2. All studies were from China and well matched in age and gender.

\section{fMRI meta-analysis of the comparison between schizophrenia patients and healthy controls}

Results of the comparison between schizophrenia patients and healthy controls from the AES-SDM analysis are summarized in Figure 2 and Table 3. The most robust findings revealed that schizophrenia patients had increased ReHo in right superior frontal gyrus and right superior temporal gyrus, whereas decreased ReHo was noted in left fusiform gyrus, left superior temporal gyrus, left postcentral gyrus, and right precentral gyrus. Jackknife sensitivity analysis showed that all these results were highly replicable. Analysis of heterogeneity suggested that two regions (right superior frontal gyrus and left fusiform gyrus) with altered ReHo had significant statistical heterogeneity among included studies. Subgroup analysis further revealed that the first episode affected the altered ReHo in right superior frontal gyrus and left superior temporal gyrus, whereas drug-naïve affected that in right superior frontal gyrus and left fusiform gyrus.

\section{fMRI meta-analysis of the comparison between unaffected relatives and healthy controls}

Results of the comparison between unaffected relatives and healthy controls from the AES-SDM analysis are summarized in Figure 3 and Table 4. The most robust findings revealed that unaffected relatives only had decreased ReHo in right insula and right superior temporal gyrus. Jackknife sensitivity and heterogeneity analyses showed that the decreased ReHo in right superior temporal gyrus was highly replicable and consistent in the included studies.

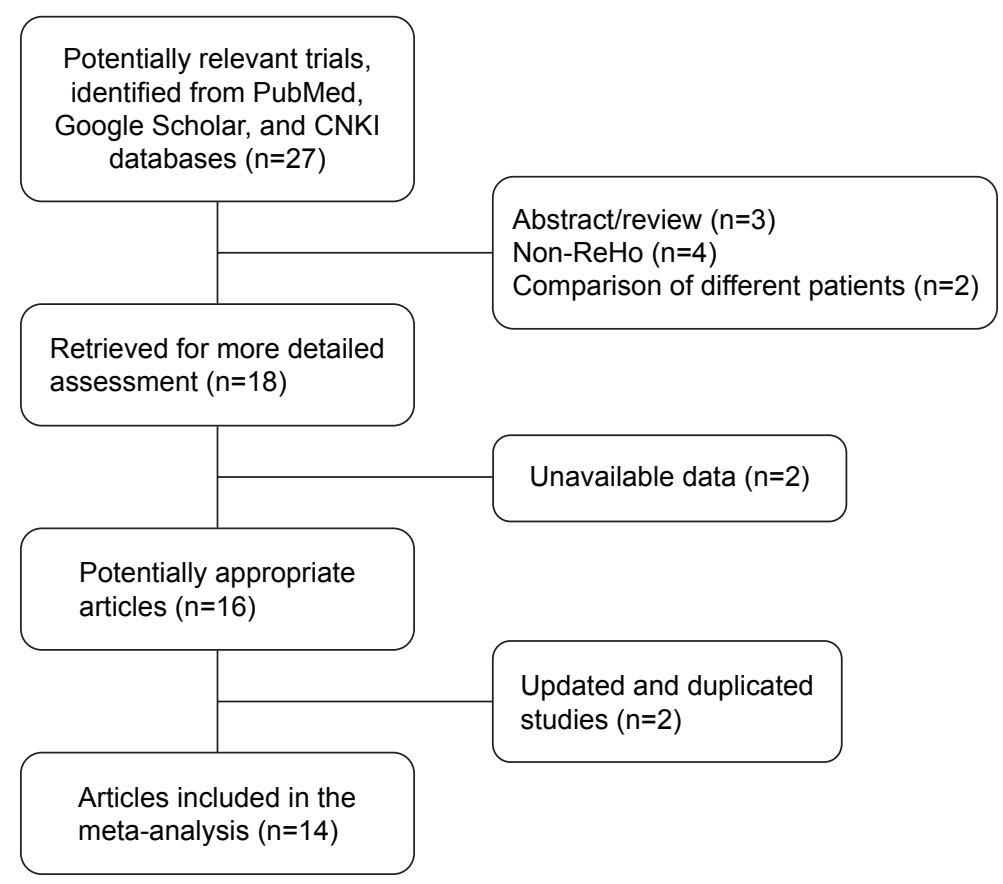

Figure I Flowchart of the selection process.

Abbreviations: CNKI, China National Knowledge Infrastructure; ReHo, regional homogeneity. 
Table I General characteristics of the included studies for meta-analysis

\begin{tabular}{|c|c|c|c|c|c|c|c|c|c|c|}
\hline \multirow[t]{2}{*}{ Authors } & \multirow[t]{2}{*}{ Year } & \multicolumn{3}{|c|}{ Sample size } & \multicolumn{2}{|c|}{ Characteristics of patients } & \multirow{2}{*}{$\begin{array}{l}\text { Mean age } \\
\text { of controls } \\
\text { (SD), years }\end{array}$} & \multirow{2}{*}{$\begin{array}{l}\text { Males in } \\
\text { controls, \%a }\end{array}$} & \multirow{2}{*}{$\begin{array}{l}\text { Duration of } \\
\text { illness (SD) }\end{array}$} & \multirow{2}{*}{$\begin{array}{l}\text { Quality } \\
\text { scores }\end{array}$} \\
\hline & & Patients & Controls & Relatives & First-episode & Drug-naïve & & & & \\
\hline Liu et $\mathrm{a}^{23}$ & 2006 & 18 & 18 & - & No & No & $24.4(3.9)$ & 50.0 & $\begin{array}{l}26.8(19.2) \\
\text { months }\end{array}$ & 20 \\
\hline Wang $^{24}$ & 2009 & 24 & 39 & 26 & No & No & $34.1(7.3)$ & 46.2 & $\begin{array}{l}\text { I33.7 (79.6) } \\
\text { months }\end{array}$ & 20 \\
\hline Jiang et $\mathrm{al}^{25}$ & 2010 & 18 & 18 & - & Yes & Partly & I6.4 (0.8) & 50.0 & $\begin{array}{l}9.6(5.9) \\
\text { months }\end{array}$ & 19 \\
\hline Liao et $\mathrm{al}^{13}$ & 2012 & - & 13 & 13 & - & - & $25.3(5.7)$ & 46.2 & - & 20 \\
\hline Chen et $\mathrm{a}^{26}$ & 2013 & 36 & 44 & - & Yes & Yes & $35.7(1.8)$ & 38.6 & NS & 17 \\
\hline Fang ${ }^{27}$ & 2013 & 20 & 20 & - & Yes & Yes & $26.0(4.7)$ & 65.0 & $\begin{array}{l}\text { I4.7 (II.4) } \\
\text { months }\end{array}$ & 18 \\
\hline Liang et $\mathrm{a}^{28}$ & 2013 & 20 & 20 & - & No & No & $29.6(7.3)$ & 100.0 & 6.I (5.8) years & 19 \\
\hline Yu et a ${ }^{29}$ & 2013 & 69 & 62 & - & No & No & $29.9(8.6)$ & NS & 7.1 (6.5) years & 17 \\
\hline Yang et $\mathrm{al}^{30}$ & 2014 & 9 & 9 & - & No & No & NS & NS & I7.4 (5.6) years & 16 \\
\hline Wang' & 2015 & 17 & 17 & - & Partly & Partly & $28.7(6.0)$ & 82.4 & $\begin{array}{l}40.3(38.0) \\
\text { months }\end{array}$ & 18 \\
\hline$X u$ et $a^{32}$ & 2015 & 24 & 21 & - & No & No & $34.1(7.6)$ & 71.4 & I3.9 (9.3) years & 18 \\
\hline Gao et al ${ }^{12}$ & 2015 & 14 & 14 & - & No & Yes & 34.9 (I3.6) & 64.3 & $9.2(8.5)$ years & 19 \\
\hline Xiong ${ }^{33}$ & 2016 & 20 & 20 & - & Yes & Yes & I $4.4(0.8)$ & 45.0 & $\begin{array}{l}8.9(3.1) \\
\text { months }\end{array}$ & 19 \\
\hline Liu et al" & 2016 & 27 & 27 & 27 & No & Partly & $27.4(7.2)$ & 66.6 & $\begin{array}{l}\text { I8.3 (15.8) } \\
\text { months }\end{array}$ & 20 \\
\hline
\end{tabular}

Notes: -indicates not involved; ${ }^{2}$ patients, controls, and relatives were matched by age and gender distribution.

Abbreviations: NS, not stated; SD, standard deviation.

\section{Discussion}

The key finding of this meta-analysis was that schizophrenia patients exhibited a significantly increased ReHo in right superior frontal gyrus and right superior temporal gyrus and decreased ReHo in left fusiform gyrus, left superior temporal gyrus, left postcentral gyrus, and right precentral gyrus, whereas unaffected relatives showed decreased ReHo in right insula and right superior temporal gyrus. These changes were highly replicable in sensitivity and subgroup analyses. Particularly, in both patients and unaffected relatives,

Table 2 Details on data acquisition and image preprocessing in resting-state of the included studies for meta-analysis

\begin{tabular}{|c|c|c|c|c|c|c|c|c|c|c|c|}
\hline Study & $\begin{array}{l}\text { Instructed } \\
\text { method }\end{array}$ & Tesla & $\begin{array}{l}\text { Eye } \\
\text { state }\end{array}$ & $\begin{array}{l}\text { TR/TE } \\
\text { (ms/ms) }\end{array}$ & $\begin{array}{l}\text { Thickness/gap } \\
(\mathrm{mm} / \mathrm{mm})\end{array}$ & $\begin{array}{l}\text { Field } \\
\text { of view } \\
(\mathbf{m m} \times \mathbf{m m})\end{array}$ & $\begin{array}{l}\text { Lasted } \\
\text { time } \\
(\mathrm{min})\end{array}$ & $\begin{array}{l}\text { Imaging } \\
\text { package }\end{array}$ & $\begin{array}{l}\text { FWHM } \\
(\mathrm{mm})\end{array}$ & Referential & Threshold \\
\hline Liu et $\mathrm{a}^{23}$ & Oral conversation & 1.5 & Closed & $2000 / 40$ & $5 / 1$ & $240 \times 240$ & 6.00 & SPM2 & 4 & $\mathrm{MNI}$ & Corrected \\
\hline Wang $^{24}$ & $\begin{array}{l}\text { Oral and informed } \\
\text { consent }\end{array}$ & 1.5 & Closed & $2000 / 40$ & $5 / 1$ & $240 \times 240$ & 5.33 & SPM2 & 6 & $\mathrm{MNI}$ & Uncorrected \\
\hline Jiang et $\mathrm{a}^{25}$ & Oral conversation & 1.5 & Closed & $2000 / 40$ & $5 / 1$ & $240 \times 240$ & NS & SPM5 & 4 & $\mathrm{MNI}$ & Corrected \\
\hline Liao et $\mathrm{al}^{13}$ & Oral conversation & 1.5 & Closed & $2000 / 40$ & $5 / 1.5$ & $240 \times 240$ & NS & SPM2 & NS & $\mathrm{MNI}$ & Uncorrected \\
\hline Chen et $\mathrm{a}^{26}$ & Oral conversation & 3.0 & Closed & $2000 / 30$ & $4 / 0$ & $192 \times 192$ & 8.00 & SPM8 & 4 & Talairach & Corrected \\
\hline Fang $^{27}$ & Oral conversation & 3.0 & Closed & $3000 / 40$ & 4/NS & $240 \times 240$ & 6.10 & SPM8 & 4 & $\mathrm{MNI}$ & Corrected \\
\hline Liang et $\mathrm{al}^{28}$ & Oral conversation & 3.0 & Closed & $3000 / 40$ & $4 / 0$ & $240 \times 240$ & 6.45 & SPM8 & NS & $\mathrm{MNI}$ & Corrected \\
\hline Yu et $\mathrm{al}^{29}$ & Oral conversation & 3.0 & Closed & $2000 / 24$ & $3 / 0$ & $256 \times 256$ & 6.00 & SPM5 & 6 & $\mathrm{MNI}$ & Corrected \\
\hline Yang et $\mathrm{al}^{30}$ & Oral conversation & 3.0 & Closed & $2200 / 35$ & $3 / 0$ & NS & 17.67 & SPM8 & 4 & $\mathrm{MNI}$ & Uncorrected \\
\hline Wang ${ }^{31}$ & Oral conversation & 1.5 & Closed & $10.5 / 2.0$ & $1.8 / 0$ & $240 \times 240$ & 14.10 & SPM8 & 4 & $\mathrm{MNI}$ & Corrected \\
\hline$X u$ et $a^{32}$ & Oral conversation & 3.0 & Closed & $3000 / 30$ & $3 / 1$ & $220 \times 220$ & NS & SPM8 & 6 & $\mathrm{MNI}$ & Uncorrected \\
\hline Gao et $\mathrm{al}^{12}$ & $\begin{array}{l}\text { Oral and informed } \\
\text { consent }\end{array}$ & 1.5 & Closed & $2000 / 40$ & $5 / 1$ & $240 \times 240$ & 8.00 & SPM8 & 6 & $\mathrm{MNI}$ & Corrected \\
\hline Xiong $^{33}$ & Oral conversation & 3.0 & Closed & $2500 / 30$ & $4 / 0$ & $240 \times 240$ & NS & SPM8 & NS & $\mathrm{MNI}$ & Corrected \\
\hline Liu et al" & Oral conversation & 1.5 & Closed & $2045 / 9.6$ & $5 / 1$ & $240 \times 240$ & 6.00 & SPM8 & 8 & $\mathrm{MNI}$ & Corrected \\
\hline
\end{tabular}

Abbreviations: NS, not stated; TR/TE, repetition time/echo time; FWHM, full width at half maximum; MNI, Montreal Neurological Institute. 


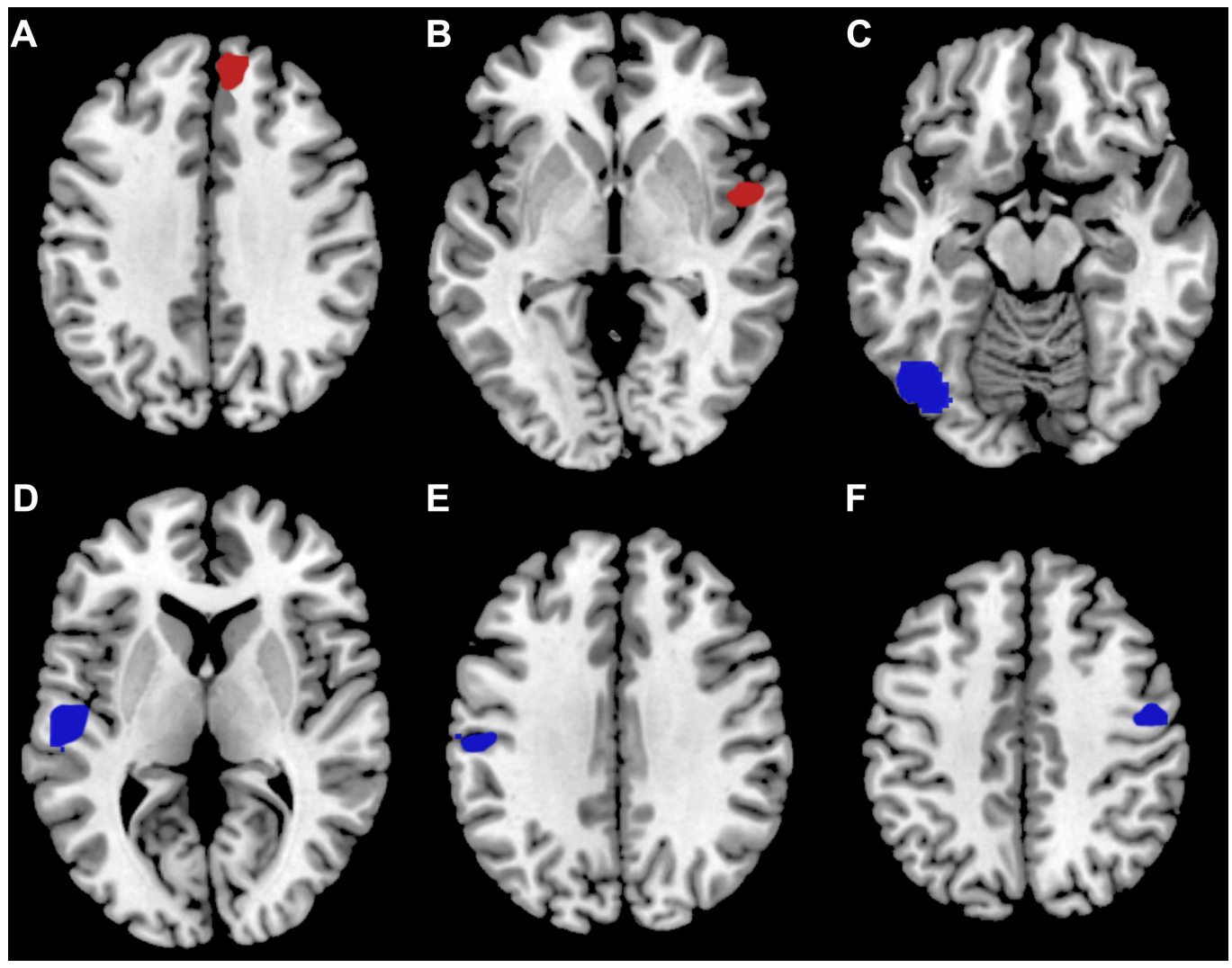

Figure 2 Meta-analytic results of ReHo studies involved in schizophrenia patients vs healthy controls.

Notes: (A) Right superior frontal gyrus; (B) right superior temporal gyrus; (C) left fusiform gyrus; (D) left superior temporal gyrus; (E) left postcentral gyrus; and (F) right precentral gyrus. Regions highlighted in red signify increased localized connectivity and regions highlighted in blue signify decreased localized connectivity in schizophrenia patients compared with healthy controls.

Abbreviation: ReHo, regional homogeneity.

Table 3 Altered ReHo in schizophrenia patients compared with healthy controls

\begin{tabular}{|c|c|c|c|c|c|c|}
\hline $\begin{array}{l}\text { Cluster } \\
\text { location }\end{array}$ & $\begin{array}{l}\text { MNI } \\
\text { coordinates } \\
(x, y, z)\end{array}$ & $z$ value & $\begin{array}{l}\text { Breakdown } \\
\text { (number of } \\
\text { voxels) }\end{array}$ & $\begin{array}{l}\text { Jackknife } \\
\text { sensitivity }\end{array}$ & Heterogeneity & $\begin{array}{l}\text { Subgroups } \\
\text { with altered } \\
\text { ReHo }\end{array}$ \\
\hline \multicolumn{7}{|l|}{ Patients $>$ controls } \\
\hline $\begin{array}{l}\text { Right superior } \\
\text { frontal gyrus }\end{array}$ & $10,44,36$ & 2.440 & $\begin{array}{l}\text { BA } 9(116) \\
\text { Corpus callosum }(90) \\
\text { BA } 32(33) \\
\text { BA } 10(19)\end{array}$ & $\begin{array}{l}12 / 13 \\
\text { Chen et } \mathrm{a}^{26}\end{array}$ & Yes & $\begin{array}{l}\text { First-episode } \\
\text { Drug-naïve }\end{array}$ \\
\hline $\begin{array}{l}\text { Right superior } \\
\text { temporal gyrus }\end{array}$ & $52,0,-2$ & 1.875 & $\begin{array}{l}\text { BA } 48(26) \\
\text { BA } 38(18)\end{array}$ & $\begin{array}{l}12 / 13 \\
\text { Liang et a }\left.\right|^{28}\end{array}$ & No & None \\
\hline \multicolumn{7}{|l|}{ Patients $<$ controls } \\
\hline $\begin{array}{l}\text { Left fusiform } \\
\text { gyrus }\end{array}$ & $-38,-78,-14$ & -2.571 & $\begin{array}{l}\text { BA I } 19(221) \\
\text { BA } 37(59)\end{array}$ & $\begin{array}{l}12 / 13 \\
\text { Yu et al }{ }^{29}\end{array}$ & Yes & Drug-naïve \\
\hline $\begin{array}{l}\text { Left superior } \\
\text { temporal gyrus }\end{array}$ & $-54,-14,6$ & -2.642 & $\begin{array}{l}\text { BA } 48(178) \\
\text { Corpus callosum (69) } \\
\text { BA } 22(29)\end{array}$ & $\begin{array}{l}1 \mathrm{I} / \mathrm{I3} \\
\text { Chen et } \mathrm{al}^{26} \text {, } \\
\text { Yu et } \mathrm{al}^{29}\end{array}$ & No & First-episode \\
\hline $\begin{array}{l}\text { Left postcentral } \\
\text { gyrus }\end{array}$ & $-54,-20,34$ & -2.379 & $\begin{array}{l}\text { BA } 48(3 \mathrm{I}) \\
\text { BA } 3(30) \\
\text { BA } 6(27) \\
\text { BA } 4(18)\end{array}$ & $\begin{array}{l}9 / / 3 \\
\text { Liu et } \mathrm{al}^{23}, \\
\text { Wang }{ }^{24}, \text { Yu et } \mathrm{al}^{29} \text {, } \\
\text { Liang et } \mathrm{a}^{28}\end{array}$ & No & None \\
\hline $\begin{array}{l}\text { Right } \\
\text { precentral } \\
\text { gyrus }\end{array}$ & $50,-8,44$ & -2.367 & $\begin{array}{l}\text { BA } 6(59) \\
\text { BA } 4(40)\end{array}$ & $\begin{array}{l}1 \mathrm{I} / 13 \\
\text { Yu et } \mathrm{al}^{29} \\
\text { Liu et } \mathrm{al}^{11}\end{array}$ & No & None \\
\hline
\end{tabular}

Abbreviations: ReHo, regional homogeneity; MNI, Montreal Neurological Institute; BA, Brodmann's area. 


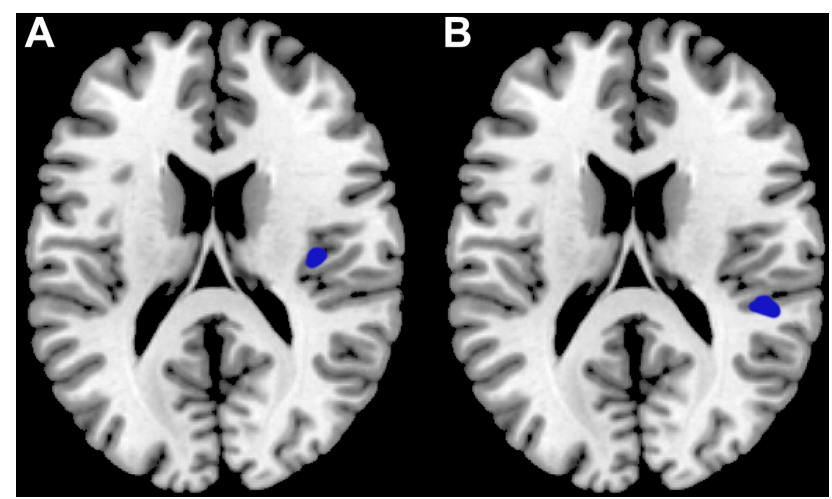

Figure 3 Meta-analytic results of ReHo studies involved in unaffected relatives vs healthy controls.

Notes: (A) Right insula and (B) right superior temporal gyrus. Regions highlighted in blue signify decreased localized connectivity in unaffected relatives compared with healthy controls.

Abbreviation: ReHo, regional homogeneity.

no consistent region was found to be changed compared with controls, indicating that abnormal localized functional connectivity may be widely and uniquely distributed in schizophrenia patients and their unaffected relatives during the resting-state. Two possible explanations may account for these results. 1) The disrupted localized connectivity in frontal and temporal cortex may be associated with the characteristic symptoms of schizophrenia, which are apparent only in patients; however, unaffected siblings are free from severe psychotic symptoms. Therefore, siblings may restrict to focal regions rather than large-scale changes in localized functional connectivity. 2) Siblings of schizophrenia patients, bearing less genetic burdens than the patient's discordant twins, only have $9 \%$ increased risk for the disease. This percentage may be inadequate for reflecting some brain abnormalities evidenced in patients. ${ }^{34,35}$

Previously, two meta-analyses of rs-fMRI studies have reported abnormal localized neural connections in schizophrenia. ${ }^{36,37} \mathrm{Kühn}_{\text {et }} \mathrm{al}^{36}$ found hypoactivation in ventromedial prefrontal cortex, left hippocampus, posterior cingulate cortex, and lower precuneus and the precuneus, as well as hyperactivation in bilateral lingual gyrus of schizophrenic patients by using activation likelihood estimation. Li et $\mathrm{al}^{37}$ performed a meta-analysis by using the Liptak-Stouffer $z$-score method to summarize stage-specific functional connectivity changes in multiple centers. Their results suggest that first-episode patients have 82 functional connections significantly differing from controls, and $94 \%$ of these are in frontal lobe meta. However, chronic-stage patients show functional connectivity differences from controls that become much more widespread (with 162 altered links), with the most prominent ones being in thalamus bilaterally (58 links) and cingulate cortex (49 links). All three meta-analyses (Kühn et al, ${ }^{36} \mathrm{Li}$ et al, ${ }^{37}$ and the present study) have revealed abnormal localized functional connections in prefrontal cortex in schizophrenia patients, but our study further demonstrated its abnormalities in unaffected relatives and the surrounding of central lobule. We also calculated the effect size of each cluster with abnormal localized neural connection and included only rs-fMRI studies performed by using ReHo analysis, which can decrease heterogeneity results from methodology and may have advantages for reflecting the localized synchronization of information processing. ${ }^{9,31}$ Our results were partially replicated in the abovementioned metaanalyses, and differences may result from our inclusion of recent studies by using ReHo analysis, the software used for the meta-analysis, and the particular parameter settings. Notably, the advantage of our meta-analysis was the systematic and comprehensive comparison among the three populations (ie, patients, unaffected relatives, and controls).

Superior temporal gyrus, a popular focus in studies on schizophrenia, may be the most interesting brain region in the present meta-analysis due to its changes in both the patients and unaffected relatives. Previous longitudinal MRI studies have reported a marked progressive decrease in superior temporal gyrus gray matter in both schizophrenia patients and their unaffected relatives. ${ }^{38-41}$ Our previous study on a single center has also demonstrated that schizophrenia patients have

Table 4 Altered ReHo in unaffected relatives compared with healthy controls

\begin{tabular}{|c|c|c|c|c|c|}
\hline Cluster location & $\begin{array}{l}\text { MNI coordinates } \\
(x, y, z)\end{array}$ & $z$ value & $\begin{array}{l}\text { Breakdown } \\
\text { (number of voxels) }\end{array}$ & $\begin{array}{l}\text { Jackknife } \\
\text { sensitivity }\end{array}$ & Heterogeneity \\
\hline \multicolumn{6}{|l|}{ Relatives $>$ controls } \\
\hline \multicolumn{6}{|l|}{ Relatives $<$ controls } \\
\hline Right insula & $36,-16,18$ & -1.087 & BA $48(43)$ & $\begin{array}{l}1 / 3 \\
\text { Wang' }{ }^{24} \text {, Liu et al" }\end{array}$ & Yes \\
\hline $\begin{array}{l}\text { Right superior } \\
\text { temporal gyrus }\end{array}$ & $44,-34,18$ & -1.086 & $\begin{array}{l}\text { BA } 48(20) \\
\text { BA } 42(11)\end{array}$ & $\begin{array}{l}2 / 3 \\
\text { Wang }^{24}\end{array}$ & No \\
\hline
\end{tabular}

Abbreviations: ReHo, regional homogeneity; MNI, Montreal Neurological Institute; BA, Brodmann's area. 
smaller gray matter volume and dysfunctional connectivity in superior temporal gyrus. ${ }^{42}$ Partly consistent with previous inclusion studies, the present meta-analysis showed that schizophrenia patients had localized connectivity decreased in left superior temporal gyrus and increased in right superior temporal gyrus, whereas unaffected relatives had decreased localized connectivity in right superior temporal gyrus. These results suggested that patients and relatives did not share the same change in these brain regions. The abnormalities in the superior temporal gyrus may play a crucial role in defects of memory, language processing, and auditory in schizophrenia, ${ }^{43}$ which may result from abnormal synaptic pruning and myelination during the neurodevelopmental process. ${ }^{44} \mathrm{Hence}$, we infer that although schizophrenia patients and their unaffected relatives had different abnormalities of localized connectivity in the superior temporal gyrus, the pathogenesis of the disorder may also be involved in abnormal changes in synaptic pruning and myelination under the different stages and environments of the neurodevelopmental process.

The surroundings of central lobule may be the second most interesting brain regions in our meta-analysis. Cortical volume reduction in the paracentral and precentral lobule of the sensory and motor networks has been reported in schizophrenia patients. ${ }^{45-47}$ Compared with healthy controls, schizophrenia patients had significantly reduced local functional connectivity density in paracentral lobule ${ }^{48,49}$ but decreased ReHo values in precentral lobule, ${ }^{23,29}$ which were consistent with the findings in the present work. Abnormalities in the surroundings of central lobule in schizophrenia patients suggested that sensorimotor cortex (SMC) may contribute to the development of the disorder. Dopaminergic terminals and receptors are known to be present in $\mathrm{SMC}^{50}$ and have the effect of reducing SMC excitability, ${ }^{51}$ suggesting that modulation may render SMC circuitry effective in processing sensory information. The abnormalities of localized connectivity in these regions in SMC may be associated with neurodevelopmental problems, such as neuronal overgrowth and a deficit in normal synaptic pruning during neurogenesis. ${ }^{44}$ From infancy, through childhood and adolescence, and into adulthood, the structural and functional maturation of neural pathways clearly connects a set of specialized brain regions related to cognitive, motor, and sensory functions. ${ }^{44}$ However, many different "connectivities" occur during neurogenesis. ${ }^{44}$ Previous studies have reported that neurodevelopment in youth is associated with increased distant connectivity and decreased local connectivity. ${ }^{52,53}$ Furthermore, considering that schizophrenia is a disorder of neurodevelopment, ${ }^{54}$ its disturbed neural connectivity is based on alterations in synaptic connection strength and indicates regional integration of information processing. ${ }^{10,55}$ Hence, the abnormal localized functional connectivity in the surroundings of central lobule within SMC may be accompanied by a neural development process and contribute to the injured information processing in schizophrenia.

Other uniquely regional changes in localized functional connectivity in patients or unaffected relatives, such as right superior frontal gyrus, left fusiform gyrus, and right insula, may also play key roles in the neuropathological endophenotypes of schizophrenia. Although the functionality of these brain regions is not fully understood, it has been linked with various neural pathways related to emotion, selfawareness, recognition, and so on, ${ }^{56-58}$ which are impaired in schizophrenia patients. ${ }^{59,60}$ In summary, our voxel-based meta-analyses identified several brain regions with abnormal localized functional connectivity in schizophrenia patients and their relatives. These abnormalities may reflect the neuropathological characteristics of this disorder and serve as a basis for establishing objective diagnostic markers for schizophrenia. Our results may also help identify the role of genetic and environmental factors in the abnormal neurodevelopment that occurs in schizophrenia.

A few limitations exist in this study. 1) We did not include studies without coordinates or with negative results. 2) Publications containing rs-fMRI data were limited, which may have led to inadequate sample size. 3) The AES-SDM software allows only peak coordinates or statistical maps to be entered as original data. Thus, the meta-analysis included only rs-fMRI studies using ReHo as a physiologically meaningful indicator of localized functional connectivity. In addition, no abnormal brain region was shared by patients and unaffected relatives. This finding may partly impede a complete understanding of the association between brain abnormalities and the pathogenesis of schizophrenia. Therefore, more studies should be conducted on structure, activity, and function in schizophrenia patients and their unaffected relatives.

\section{Conclusion}

Despite these limitations, the present meta-analysis revealed that schizophrenia patients and their unaffected relatives had extensive abnormal localized connectivity in cerebrum, especially in superior temporal gyrus, which were the potential diagnostic markers and expounded the pathophysiological hypothesis for the disorder.

\section{Acknowledgments}

The authors thank Yi He (Chengdu FX Biotechnology Co., Ltd.) for assistance in the methodological preparation. This 
study was funded by the National Science and Technology Ministry (2012BAI01B02).

\section{Disclosure}

The authors report no conflicts of interest in this work.

\section{References}

1. Wang S, Li W, Zhang H, et al. Association of microRNA137 gene polymorphisms with age at onset and positive symptoms of schizophrenia in a Han Chinese population. Int J Psychiatry Med. 2014;47(2): $153-168$.

2. Wang S, Li WQ, Zhao JY, et al. Association of estrogen receptor alpha gene polymorphism with age at onset, general psychopathology symptoms, and therapeutic effect of schizophrenia. Behav Brain Funct. 2013;9:12.

3. Reid MA, Stoeckel LE, White DM, et al. Assessments of function and biochemistry of the anterior cingulate cortex in schizophrenia. Biol Psychiatry. 2010;68(7):625-633.

4. Guo WB, Hu MR, Fan XD, et al. Decreased gray matter volume in the left middle temporal gyrus as a candidate biomarker for schizophrenia: a study of drug naive, first-episode schizophrenia patients and unaffected siblings. Schizophr Res. 2014;159(1):43-50.

5. Glahn DC, Thompson PM, Blangero J. Neuroimaging endophenotypes: strategies for finding genes influencing brain structure and function. Hum Brain Mapp. 2007;28(6):488-501.

6. Rose EJ, Donohoe G. Brain vs behavior: an effect size comparison of neuroimaging and cognitive studies of genetic risk for schizophrenia. Schizophr Bull. 2013;39(3):518-526.

7. Whitfield-Gabrieli S, Thermenos HW, Milanovic S, et al. Hyperactivity and hyperconnectivity of the default network in schizophrenia and in first-degree relatives persons with schizophrenia. Proc Natl Acad Sci US A. 2009;106(4):1279-1284.

8. Guo WB, Liu F, Xue ZM, et al. Abnormal neural activities in first-episode, treatment-naive, short-illness-duration, and treatmentresponse patients with major depressive disorder: a resting-state fMRI study. J Affect Disord. 2011;135(1-3):326-331.

9. Zang Y, Jiang T, Lu Y, He Y, Tian L. Regional homogeneity approach to fMRI data analysis. Neuroimage. 2004;22(1):394-400.

10. Zuo XN, Xu T, Jiang L, et al. Toward reliable characterization of functional homogeneity in the human brain: preprocessing, scan duration, imaging resolution and computational space. Neuroimage. 2013;65: 374-386.

11. Liu C, Xue Z, Palaniyappan L, et al. Abnormally increased and incoherent resting-state activity is shared between patients with schizophrenia and their unaffected siblings. Schizophr Res. 2016;171(1-3):158-165.

12. Gao B, Wang Y, Liu W, et al. Spontaneous activity associated with delusions of schizophrenia in the left medial superior frontal gyrus: a resting-state fMRI study. PLoS One. 2015;10(7):e0133766.

13. Liao H, Wang L, Zhou B, et al. A resting-state functional magnetic resonance imaging study on the first-degree relatives of persons with schizophrenia. Brain Imaging Behav. 2012;6(3):397-403.

14. Moher D, Shamseer L, Clarke M, et al. Preferred reporting items for systematic review and meta-analysis protocols (PRISMA-P) 2015 statement. Syst Rev. 2015;4:1.

15. Brambilla P, Hardan A, di Nemi SU, Perez J, Soares JC, Barale F. Brain anatomy and development in autism: review of structural MRI studies. Brain Res Bull. 2003;61(6):557-569.

16. Shepherd AM, Matheson SL, Laurens KR, Carr VJ, Green MJ. Systematic meta-analysis of insula volume in schizophrenia. Biol Psychiatry. 2012;72(9):775-784.

17. Iwabuchi SJ, Krishnadas R, Li C, Auer DP, Radua J, Palaniyappan L. Localized connectivity in depression: a meta-analysis of resting state functional imaging studies. Neurosci Biobehav Rev. 2015;51:77-86.
18. Zhao YJ, Du MY, Huang XQ, et al. Brain grey matter abnormalities in medication-free patients with major depressive disorder: a metaanalysis. Psychol Med. 2014;44(14):2927-2937.

19. Radua J, van den Heuvel OA, Surguladze S, Mataix-Cols D. Meta-analytical comparison of voxel-based morphometry studies in obsessive-compulsive disorder vs other anxiety disorders. Arch Gen Psychiatry. 2010;67(7):701-711.

20. Cooper D, Barker V, Radua J, Fusar-Poli P, Lawrie SM. Multimodal voxel-based meta-analysis of structural and functional magnetic resonance imaging studies in those at elevated genetic risk of developing schizophrenia. Psychiatry Res. 2014;221(1):69-77.

21. Radua J, Mataix-Cols D, Phillips ML, et al. A new meta-analytic method for neuroimaging studies that combines reported peak coordinates and statistical parametric maps. Eur Psychiatry. 2012;27(8):605-611.

22. Radua J, Mataix-Cols D. Voxel-wise meta-analysis of grey matter changes in obsessive-compulsive disorder. Br J Psychiatry. 2009;195(5): 393-402.

23. Liu H, Liu Z, Liang M, et al. Decreased regional homogeneity in schizophrenia: a resting state functional magnetic resonance imaging study. Neuroreport. 2006;17(1):19-22.

24. Wang J. The Analysis of Clinical Characters and MRI Study on Brain Functional and Structural Changes in Patients with Treatment-Resistant Schizophrenia and Their Relatives. Changsha: Second Xiangya Hospital, Central South University; 2009.

25. Jiang S, Zhou B, Liao Y, et al. Primary study of resting state functional magnetic resonance imaging in early onset schizophrenia using ReHo. Zhong Nan Da Xue Xue Bao Yi Xue Ban. 2010;35(9):947-951.

26. Chen J, Xu Y, Zhang K, et al. Comparative study of regional homogeneity in schizophrenia and major depressive disorder. Am J Med Genet $B$ Neuropsychiatr Genet. 2013;162B(1):36-43.

27. Fang L. Resting-State Functional Magnetic Resonance Imaging Study of Brain Function in the First-Episode Paranoid-Type Schizophrenia Patients. Nanjing: The Fourth School of Clinical Medicine, Nanjing Medical University; 2013.

28. Liang J, Xie S, Du J, Yan W. Regional homogeneity of functional magnetic resonance imaging in male schizophrenia patients with delusion symptoms. J Clin Psychiatry. 2013;23(6):379-382.

29. Yu R, Hsieh MH, Wang HL, et al. Frequency dependent alterations in regional homogeneity of baseline brain activity in schizophrenia. PLoS One. 2013;8(3):e57516.

30. Yang F, Yang T, Kang C, et al. The change of resting state functional magnetic resonance imaging in chronic schizophrenia patients combined risperidone treatment. J Clin Psychiatry. 2014;24(2):73-76.

31. Wang W. The Follow Up Study of Regional Homogeneity of Brain Rest State Functional Schizophrenia Treated with Paliperidone Injection for 3 Months. Kunming: The First Affiliated Hospital, Kunming Medical University; 2015.

32. Xu X, Shi X, Peng Z, Wang X, Sun J, Yao H. A study of resting-state functional magnetic resonance imaging in treatment-refractory schizophrenia patients using regional homogeneity. J Clin Psychiatry. 2015; 25(6):377-380.

33. Xiong Y. Resting State fMRI Study of Amplitude of Low-Frequency Fluctuation and Regional Homogeneity in Early Onset Schizophrenia. Taiyuan: First Hospital, Sanxi Medical University; 2016.

34. Moran ME, Hulshoff Pol H, Gogtay N. A family affair: brain abnormalities in siblings of patients with schizophrenia. Brain. 2013;136(pt 11): 3215-3226.

35. Chang X, Shen H, Wang L, et al. Altered default mode and frontoparietal network subsystems in patients with schizophrenia and their unaffected siblings. Brain Res. 2014;8:87-99.

36. Kuhn S, Gallinat J. Resting-state brain activity in schizophrenia and major depression: a quantitative meta-analysis. Schizophr Bull. 2013; 39(2):358-365.

37. Li T, Wang Q, Zhang J, et al. Brain-wide analysis of functional connectivity in first-episode and chronic stages of schizophrenia. Schizophr Bull. 2016. Epub 2016 Jul 21. 
38. Kasai K, Shenton ME, Salisbury DF, et al. Progressive decrease of left superior temporal gyrus gray matter volume in patients with firstepisode schizophrenia. Am J Psychiatry. 2003;160(1):156-164.

39. Takahashi T, Wood SJ, Yung AR, et al. Progressive gray matter reduction of the superior temporal gyrus during transition to psychosis. Arch Gen Psychiatry. 2009;66(4):366-376.

40. Borgwardt SJ, Riecher-Rössler A, Dazzan P, et al. Regional gray matter volume abnormalities in the at risk mental state. Biol Psychiatry. 2007; 61(10):1148-1156.

41. Rajarethinam R, Sahni S, Rosenberg DR, Keshavan MS. Reduced superior temporal gyrus volume in young offspring of patients with schizophrenia. Am J Psychiatry. 2004;161(6):1121-1124.

42. Zhang Y, Zheng J, Fan X, et al. Dysfunctional resting-state connectivities of brain regions with structural deficits in drug-naive first-episode schizophrenia adolescents. Schizophr Res. 2015;168(1-2):353-359.

43. Rajarethinam RP, DeQuardo JR, Nalepa R, Tandon R. Superior temporal gyrus in schizophrenia: a volumetric magnetic resonance imaging study. Schizophr Res. 2000;41(2):303-312.

44. Paus T, Keshavan M, Giedd JN. Why do many psychiatric disorders emerge during adolescence? Nat Rev Neurosci. 2008;9(12):947-957.

45. Hirjak D, Wolf RC, Stieltjes B, et al. Cortical signature of neurological soft signs in recent onset schizophrenia. Brain Topogr. 2014;27(2): 296-306.

46. Zhou SY, Suzuki M, Hagino H, et al. Volumetric analysis of sulci/ gyri-defined in vivo frontal lobe regions in schizophrenia: precentral gyrus, cingulate gyrus, and prefrontal region. Psychiatry Res. 2005; 139(2):127-139.

47. Borgwardt SJ, Picchioni MM, Ettinger U, Toulopoulou T, Murray R, McGuire PK. Regional gray matter volume in monozygotic twins concordant and discordant for schizophrenia. Biol Psychiatry. 2010;67(10): 956-964.

48. Lui S, Yao L, Xiao Y, et al. Resting-state brain function in schizophrenia and psychotic bipolar probands and their first-degree relatives. Psychol Med. 2015;45(1):97-108.
49. Chen X, Duan M, Xie Q, et al. Functional disconnection between the visual cortex and the sensorimotor cortex suggests a potential mechanism for self-disorder in schizophrenia. Schizophr Res. 2015;166(1-3): $151-157$.

50. Lewis DA, Campbell MJ, Foote SL, Goldstein M, Morrison JH. The distribution of tyrosine hydroxylase-immunoreactive fibers in primate neocortex is widespread but regionally specific. J Neurosci. 1987;7(1): 279-290.

51. Hosp JA, Hertler B, Atiemo CO, Luft AR. Dopaminergic modulation of receptive fields in rat sensorimotor cortex. Neuroimage. 2011;54(1): 154-160.

52. Satterthwaite TD, Wolf DH, Loughead J, et al. Impact of in-scanner head motion on multiple measures of functional connectivity: relevance for studies of neurodevelopment in youth. Neuroimage. 2012;60(1): 623-632.

53. Dosenbach NUF, Nardos B, Cohen AL, et al. Prediction of individual brain maturity using fMRI. Science. 2010;329(5997):1358-1361.

54. Lewis DA, Levitt P. Schizophrenia as a disorder of neurodevelopment. Annu Rev Neurosci. 2002;25:409-432.

55. Karlsgodt KH, Sun D, Jimenez AM, et al. Developmental disruptions in neural connectivity in the pathophysiology of schizophrenia. Dev Psychopathol. 2008;20(4):1297-1327.

56. Goldberg II, Harel M, Malach R. When the brain loses its self: prefrontal inactivation during sensorimotor processing. Neuron. 2006;50(2) 329-339.

57. Beauregard M, Levesque J, Bourgouin P. Neural correlates of conscious self-regulation of emotion. J Neurosci. 2001;21(18):RC165.

58. Berthier M, Starkstein S, Leiguarda R. Behavioral effects of damage to the right insula and surrounding regions. Cortex. 1987;23(4):673-678.

59. Crow TJ. Positive and negative schizophrenia symptoms and the role of dopamine. Br J Psychiatry. 1981;139:251-254.

60. Ritsner M, Ben-Avi I, Ponizovsky A, Timinsky I, Bistrov E, Modai I. Quality of life and coping with schizophrenia symptoms. Qual Life Res. 2003;12(1):1-9.
Neuropsychiatric Disease and Treatment

\section{Publish your work in this journal}

Neuropsychiatric Disease and Treatment is an international, peerreviewed journal of clinical therapeutics and pharmacology focusing on concise rapid reporting of clinical or pre-clinical studies on a range of neuropsychiatric and neurological disorders. This journal is indexed on PubMed Central, the 'PsycINFO' database and CAS,

\section{Dovepress}

and is the official journal of The International Neuropsychiatric Association (INA). The manuscript management system is completely online and includes a very quick and fair peer-review system, which is all easy to use. Visit http://www.dovepress.com/testimonials.php to read real quotes from published authors. 